\title{
Thinking of the advance of software development method in the future
}

\author{
$\mathrm{Yu}$ Gao \\ Department of Computer,Zhejiang Ocean \\ University \\ Zhoushan, Zhejiang, P.R.China 316000 \\ gaoyu@zjou.edu.cn
}

\author{
Zhen-Bo Bi \\ Department of Computer,Zhejiang Ocean \\ University, \\ Zhoushan, Zhejiang, P.R.China 316000 \\ bzb136@sina.com
}

\begin{abstract}
Continuity of software development activities determines that software development method is bound to progress. For the future advance of development of software development methodology, six important factors are put forward. The six factors are: software development techniques and tools of, software development efficiency and quality, flexibility of software development methods, applicable scopes of software development methods, network application and the innovation of ways of thinking. The six factors are also six jobs that people should do well. For improving the existing software development methods and creating the new software development methods, these six work has an important significance.
\end{abstract}

Keywords-component; software development method; future development; technology and tool; efficiency and quality; flexibility; applicable scope; network; mode of thinking

\section{INTRODUCTION}

Since the emergence of software development activities, software development methods began to be studied. A variety of software development methods have been put forward successively [1]. For example, there are structured software development method, object-oriented software development methods, component-based software development method, formal software development method, agile software development methods, Agent-oriented software development method [2], and so on. In software development activities of past, with the help of these software development methods, a good deal of software has successfully developed.

The history of software development activities is also the history researching software development methods, and it is also the history of evolution of software development methods. Up to this day, due to the software development activities are continuing, new problems still may appear, so the software development method does not stop the pace of development and change. Therefore, for the future development of software development methods, people must think.

For the future development of the software development method, we have pondered over six key factors closely related as followed below.

\section{TECHNOLOGY AND TOOL TO SOFTWARE DEVELOPMENT METHOD}

Techniques and tools promoted development of software development method. Review the history of software development, when the new development technologies and tools emerge, based on the new development technique and tool, new software development method will appear.

Formation of structured software development method is in this way. In the 1960's, structured programming techniques began to appear, to the 1970's, it was perfect. For the structured software development methods, structured programming techniques laid its main foundation. The development tools in the subsequent emergence (such as: data flow diagrams, ER diagram, HIPO diagram, flowchart or box diagram or PAD figure, $\mathrm{C}$ language, or other structured programming language) make an important contribution to improve the structured software development method. Based on the structured programming techniques and these development tools, structured software development method evolved and is widely used. As we are now well known: It uses data flow diagrams and ER diagrams and other tools, while structural analysis. It uses tools of HIPO diagram, flowchart or box diagram or PAD diagram, while structural design. It uses the $C$ language (or other structured programming language), while programming. Therefore, we can say: because structured programming techniques and these tools have appeared, then structured software development methods have appeared. If there are not structured programming techniques and these development tools, then there is not structured software development method.

Formation of object-oriented software development method is also in this way. In 1967, concept of "class" and "object" appeared in the Simula language. In 1972, concept of "class" and "object" were strengthened in the Smalltalk language. In 1980, Smalltalk language is perfect. In 1983, C ++ language has been officially launched, since then, concept of "class" and "object" attracted extensive attention, object-oriented programming techniques evolved. On this basis, object-oriented software development methods gradually develop. In 1997, UML1.0 version officially released, it is mark, object-oriented software development methods matured. Currently, object-oriented software development method is widely used. During object-oriented analysis and design, it uses the graphical tools of UML and other tools. During object-oriented programming, it uses C++ or Java (or other object-oriented programming language). Therefore, we can say: because object-oriented programming techniques and UML tool have appeared, then object-oriented software development methods have appeared. If there are not object-oriented programming 
techniques and UML tool, then there is not object-oriented software development method.

Formation of software development methods based reuse technology is also in this way. In the early software development activities, reuse technology is immature, and the scope of reuse is limited in the level of source code. For example, in the $\mathrm{C}$ language compiling system, a variety of function was reuse by people in the "standard function library". To 1990's, "software components" were accumulated more and more through software development activities, scope of software reuse gradually increasing. "Software components" not only include the source code, development plan, architecture, design proposal, user interface, technical documentation and other materials are also included. Reuse technology gradually develop. Based on reuse technology, to use "software component library", some new software development methods have emerged, such as component-based software development method [3], such as COTS-based software development method [4], and so on.

According to the above description, software development techniques and tools are closely related to development of software development methods. For the future development of the software development method, software development techniques and tools is an important factor. Different technologies and tools support different software development methods.

Therefore, considering the future development of software development method, software development techniques and tools should be one of the content while is researched emphatically. Through research, target is to put forward new and better software development techniques and tools. Based on the new development technology and tools, people can build new software development methods.

\section{EFFICIENCY AND QUALITY TO SOFTWARE DEVELOPMENT METHOD}

For the future development of the software development method, efficiency and quality of software development is an important factor. Over the years, in order to improve the efficiency and quality of development, people have been looking for better software development methods.

In order to improve development efficiency and quality, an important step is the application of mathematical knowledge in the development process, on this basis, a number of formal software development methods have been introduced. Although the formal software development methods can eliminate ambiguity, and can improve software quality, however, a number of conditions restricted its application. If the software is relatively large, or the mathematical basis of the developer is poor, then the application of the method is more difficult. Therefore, over the years, formal software development method is difficult to spread. For formal software development method, theoretical research is more, but practical application is small.

In order to improve development efficiency and quality, an important step is to put forward software development methods based on reuse technology. When using the software development methods, "software component" is reused largely. Because quality of "software component" is guaranteed, so the efficiency and quality of development can improve. However, such software development methods rely on the "software component library". "Software Component Library" must have enough "software components", so the efficiency and quality of development can improve. Currently, for any development field, the quantity of "software components" is not enough in "software component library", and "software component library" lack versatility. When developing software, "software component" from the "software component library" is just a small part, and most of the "software component" still needs re-developing. "Software component" using in a particular field can not be directly used for other field. Therefore, the development efficiency can not be improved significantly.

To consider the future development of the software development methods, to improve efficiency and quality of development are very important, following things need to be done well.

First. According to the previous description, formal software development method needs be further improved. Goal is to achieve mass level (that is to say, developers are not hindered for profound mathematical knowledge), and not limit to the size of software. According to the current situation, the realization of above goal is difficult. However, above goals must be achieved, such, formal software development method can do something in improving efficiency and quality of development, and otherwise its development is difficult.

Second. According to the previous description, software reuse technology needs be further improved. To do the two things: One thing is that amount of "software components" should be enough in "software component library", it can basically meet the needs of software development in the certain field. Another thing is to enhance universal and applicability of "software components". When developing software, "software component" can be directly reused, or "software components" can be reused after it is modified slightly. After doing such two things, on the problem of improving efficiency and quality of development, it can really play a role that software development method based on reuse technology.

Third. Do not be limited to formalization method and reuse technology, people should look for other way in order to improve efficiency and quality of development.

\section{FleXiBILITy of Software DeVElopment Methods}

For most of today's software development methods, its content is fixed. That is to say, its development process has been fixed already, and techniques and tools of development have been fixed already. In the development of software, developers are required to operate according to fixed content. In fact, in recent years, variable factor increased gradually in development process. Face the circumstance of constantly changing, if to follow content of software development method is rigid, then normal progress of development work may be affected, even the development may fail.

Therefore, considering the future development of software development methods, in order to meet changing circumstances in development process, to effectively respond 
to a variety of variables, software development methodology must possess flexibility.

The flexibility of software development method is to allow that content of software development method is not fixed. As the situation evolves, its content can be appropriately changed.

In this study, the designers of RUP method [5] made a good try. RUP method allows to be cut. After cutting, the developer obtains a specific shape of RUP method. For example, literature [6] describes a situation of cutting RUP method. After RUP method was cut, it is suitable for software development of building enterprise information systems. Such, RUP method possessed flexibility.

Same, in this study, the designers of Agile method [7] made a good try. One of the values of Agile method is "emphasis on changes in response to even blindly follow plan". According to the value, when the development, developers should adapt to change, and make the right response to change of situation, and may not follow the original development plan. Such, Agile method possessed flexibility.

Although the RUP method allows to be cut, but after cutting, its contents remain is still fixed. In the development process, if the situation has changed, then how to do ? Although the Agile method pay attention to "responding the change", however, there are diverse changes, how do developers respond ? Clearly, there is a problem of bring into play the developer's initiative and creativity. However, on this issue, RUP method and Agile method are all not explored in depth.

From the perspective of flexibility, to think about the future development of software development method, we think that RUP method and agile method need to be further improved. After RUP method to be cut, when circumstance change in development process, developers need to determine the solution of response to the change. That is to say, developer's initiative and creativity need to be bring into play. For Agile method, when responding the change, developers need to determine the solution of response to the change. Developer's initiative and creativity need also to be bring into play.

To consider the future development of the software development methods, in order to improve flexibility, following things need to be done well.

First, like RUP and Agile methods, when building new software development methods, beneficial rules to flexibility should be draft.

Second, in allusion to problem bring into play developer's initiative and creativity, people should draft specific measure. In the development process, when the situation changes, to the method of development activity the developer can decide.

In order to play the developer's initiative and creativity, in some developed steps, the content of software development method should be only frame (not limited to the specific details of development, development techniques and tools). Or, to prepare a variety of plans, depending on the circumstances, developers choose in these plans.

In addition, which step do the flexibility should be reflected in development activities? How much degree do flexibility should be? In the future, when building a new software development method, people also need to think these problems.

\section{APPliCABle SCOPE OF SOFTWARE DeVElOPMENT METHOD}

For a long time, when building a software development method, it is hoped that applicable scope of software development methods is broad. For a certain software development method, people want that it is fully applicable under any conditions, for any field, to solve any problems. To present familiar software development method, their applicable scope is all broad.

Is it good that applicable scope of software development method is broad? We have to analyze.

If applicable scope of a software development method is wide, then the advantage are obvious, can not say here. However, it is bound that pertinence of the software development method is weak. Because it needs to meet a variety of conditions, and it needs to suit a variety of requirements, so it can not be detailed that its development process, techniques and tools. When we use this software development method in developing certain software, because of its development process, techniques and tools are not detailed, so we need that its development process, techniques and tools are all detailed. This is equivalent to reformulate a new software development method to contrapose that problem, such a lot of time and effort should be spend.

In addition, any field has its specificity, when developing software in a particular field, developer must take into account its specificity. That is to say, in order to ensure successful software development in that field, software development method should be consistent with its special requirements. Pertinence of software development methods to meet such requirements is very good. Or other, it is dedicated software development method in such field. However, it can not be broad, and its applicable scope is only in such field. For example, [8] introduced a special software development method to use for embedded software, its applicable scope is only the embedded software, and other development issues can not be resolved using this method.

Broad scope and strong pertinence is a contradiction. To consider the future development of the software development methods, when building new software development method, we should not only build some of software development methods of the broad scope, but also to build some of software development methods of strong pertinence.

The current situation is: the fields to need developing software are more and more, each field is different to the other field, nature and scale of software is different. Therefore, to build a omnipotent software development method is impossible. So, in the future, to contrapose a certain software development problems in a certain field, to build software development method of strong pertinence should be emphasis of research. 


\section{Software Development Method and EfFect of NETWORK}

Early on, the network is underdeveloped, so the network is not affected for software development. As the development of network, the relationship between software development and network gradually close up, therefore, when to study the future development of software development methods, network is an important factor that must be considered.

Popularity of the network has changed the traditional form of software development. Networks provide a new software development environment. In the case of the network does not exist, in order to communicate with each other easily, all developers of development team must locate in the same location, for example, they locate in the same office or adjacent office. If a developer left this location, the development will be affected. Application of network technology has changed this situation. Forms of development can not be restricted about geographical position, developers can use network to exchange development information. Members of development team can not in the same location. They use the network at the same time to participate in the development work. As long as the network is connected, development work will not be affected.

In the software development method of past, network's effect is not reflected. So far, for the relationship between the network and developing software, for software development method of web-based technology, research article is few. In [9], a in software development method of web-based information sharing has been introduced. In [10], a software development method of SOA-based has been introduced. From these research papers we have seen that the research has not yet under way.

To consider the future development of software development method, the network's effect in software development need to be played, and people need to build software development method of web-based technology. To achieve this purpose, the following things should be done well.

First, network development platform need to be build. When developing software, people can use the network development platform. Whether or not in the same location, all members can exchange information, and can analyze and design the target software system at the same time.

Second, "web-based software component library" need to be build. Whether or not in the same location, all members of development team can use the "web-based software component library". Through the network, they can add software components to the "web-based software component library", and can reuse software component of the "software component library".

Third, based on "network development platform" and "web-based software component library ", we research and build new software development methods of web-based technology.

\section{SOFTWARE DEVELOPMENT METHOD AND INNOVATING OF THINKING MODE}

To analyze various existing software development method, we can see that different software development methods reflect the different mode of thinking. Or other, according to different mode of thinking, different software development methods have been introduced.

In software development history, after a new mode of thinking appear (after thinking innovate) , the new software development method will follow.

For example, before formal software development methods appear, when describing software analysis and design process, a variety of software development methods use natural language. This traditional mode of thinking may lead to ambiguity. For insufficient of traditional mode of thinking, people put forward a new mode of thinking. New mode of thinking uses mathematical language to describe the software analysis and design process. Such ambiguity can be avoided, and can improve software quality. With this new mode of thinking, some of formal software development method produced, such as Clean Room method, and so on.

For example, traditional software development methods emphasize the control of development process, also emphasize predictability and visibility of development process. Therefore traditional software development methods require a lot of intermediate products (process documentation materials). However, for possible changes, it rarely considered. Such to improve the development efficiency is difficult. For the shortcomings of traditional software development methods, members of Agile Alliance put forward a new mode of thinking, This new mode of thinking emphasize to adapt the change and to simplify development process, and advocate people-oriented development process, its purpose is to improve development efficiency. Under the guidance of this new mode of thinking, some of the agile software development methods emerge, such as XP, and so on.

In order to solve the existing problems in software development activities, people must use the new mode of thinking look at software development activities (thinking innovation). The history fact of software development has proven this point.

To consider the future development of software development method, mode of thinking must continue to innovate. At present, in software development activities, there are some problems that plague us, such as software development quality problems, such as software development efficiency problems, and so on. In the future there will be new problems. We need mode of thinking to innovate, and put forward new mode of thinking. Under the guidance of new mode of thinking, we build new software development method, and solve the new problem in software development activity.

\section{CONCLUSION}

With the deepening of software development activities, software development methodology is bound to develop. The six factors are also six tasks that people should do, in order to 
develop theory of software development methodology. The future development of the software development methods, both includes to improve and to perfect existing software development methods, but also includes building new software development methods. If we do well the six works, then the existing software development methods can be improved and perfected, and new software development method can be built, and level of developing software will be raise to a new extent.

\section{ACKNOWLEDGMENT}

This research is sponsored by Natural Science Foundation of Zhejiang(No. LY12F02021).

\section{REFERENCES}

[1] Roger S Pressman, Software Engineering: A Practitioner's Approach. (6th Edition), McGraw-Hill, New York, 2005.

[2] Yan Qi, etc., "A Survey and Evaluation of Agent-Oriented Software Development Methodologies", COMPUTER ENGINEERING \& SCIENCE, 2005, vol.27, pp.67-69.
[3] WAN Lin-rui, HU Hong, SUN Hong-xing, "Researches on Component Oriented Software Development Methodology", MINIMICRO SYSTEMS, 2003, vol.24, pp.365-370.

[4] QU Xi-long, "Research for Development of COTS-based Software System”, Application Research of Computers, 2005, vol.23, pp.43-45.

[5] Jacobson I, Booch G, Rumbaugh J, The Unified Software Development Process (Third Edition), Addison Wesley,2004.

[6] LU Yongzhong, etc., "Study on Applying RUP to Develop Information System of Construction Enterprise", Computer Engineering, 2006, vol.32, pp.259-261.

[7] Zhang JingZhou, Qian LeQiu, and Zhu SanYuan, "An Overview of Agile Methodology”, Computer Applications and Software, 2002, vol.6, pp.1-9.

[8] YAO Yizhan, ZHANG Gang, "VOV Software Development Methodology”, Computer Engineering, 2005, vol.31, pp.75-77+93

[9] DU Wen lian, YANG Li, ZHUO Chun, "Research and application of network information resource sharing-based software development method”, Application Research of Computers, 2008, vol.25, pp.2713$2717+2745$.

[10] ]WEI Dong, CHEN Xiao-jiang, FANG DING-yi, "Research on the Software Development Based on SOA", Microelectronics \& Computer, 2005, vol.22, pp.73-76. 